\title{
El concreto elaborado con aguas lluvia como aporte ambiental desde la construcción
}

\author{
Concrete prepared with rain water as an environmental \\ contribution from the construction
}

O concreto elaborado com águas de chuva como aporte
ambiental desde a construção

Fecha de recepción: 08 de julio de 2015

Fecha de aprobación: 17 de noviembre de 2015

Carlos Mauricio Bedoya-Montoya*

Carlos Andrés Medina-Restrepo**

\section{Resumen}

Se aborda la confección del concreto con aguas lluvia y se compara su desempeño con respecto a los elaborados con agua potabilizada, empleada convencionalmente. Las muestras confeccionadas se estudiaron en cuanto a la resistencia al esfuerzo a compresión a edades de 1, 3, 7, 14, 28, 56 y 90 días, analizando también su microestructura por medio de microscopía de barrido electrónico (SEM) y caracterizando el agua involucrada mediante laboratorio certificado. Luego de analizar los resultados, se ve factible que la confección del concreto puede hacerse de modo más racional en cuanto a la utilización del recurso hídrico, sin sacrificar sus cualidades mecánicas, estéticas y de estabilidad. A su vez, se contempla la posibilidad de establecer una práctica que pueda ser replicable en el contexto colombiano.

Palabras clave: Construcción sostenible, Concreto con agua lluvia.

\section{Abstract}

This article describes the preparation of concrete using rain water and its performance compared with those elaborated with conventional drinking water. The samples made were studied in terms of resistance to compressive stress at ages of 1, 3, 7, 14, 28, 56 and 90 days, also analyzing their microstructure by electronic microscopy scanning (EMS) and characterizing the water involved by a certified laboratory. After analyzing the results, it looks likely that the construction of the concrete can be done in a more thoughtful way regarding

* M.Sc. Universidad Nacional de Colombia (Medellín-Antioquia, Colombia). cmbedoya@unal.edu.co.

** M.Sc. Institución Universitaria Colegio Mayor de Antioquia (Medellín-Antioquia, Colombia). carlos.medina@colmayor.edu.co. 
the use of water resources, without sacrificing its mechanical, stability and aesthetic qualities. In turn, may be thought the possibility to establish a practice that can be replicated in the Colombian context.

Keywords: Sustainable construction, Rain water, Concrete.

\section{Resumo}

Aborda-se a confecção do concreto com águas de chuva e compara-se seu desempenho com respeito aos elaborados com água tratada, empregada convencionalmente. As amostras confeccionadas se estudaram em quanto à resistência ao esforço a compressão a idades de 1, 3, 7, 14, 28, 56 e 90 dias, analisando também sua microestrutura por meio de microscopia de varredura eletrônica (SEM) e caracterizando a água utilizada mediante laboratório certificado. Depois de analisar os resultados, é factível que a confecção do concreto pode fazer-se de modo mais racional em quanto à utilização do recurso hídrico, sem sacrificar suas qualidades mecânicas, estéticas e de estabilidade. Por sua vez, se contempla a possibilidade de estabelecer uma prática que possa ser replicável no contexto colombiano.

Palavras chave: Construção sustentável, Concreto com água de chuva.

Cómo citar este artículo:

[1] C. M. Bedoya-Montoya \& C. A. Medina-Restrepo, "El concreto elaborado con aguas lluvia como aporte ambiental desde la construcción”, Fac. Ing., vol. 25 (41), pp. 31-39, ene.-abr. 2016. 


\section{INTRODUCCIÓN}

El agua empleada en la confección de un concreto debe cumplir con parámetros físico-químicos que, para el caso colombiano, están contemplados en la norma técnica NTC 3459. Dicha norma $-y$ otras referencias bibliográficas sobre tecnología del concreto- establece en uno de sus apartes que el agua apta para el consumo humano puede ser utilizada para elaborar mezclas de concreto [1]. Es una práctica común, entonces, utilizar el agua potabilizada para fabricar concreto, sin ninguna verificación previa, suponiendo que toda agua que es potabilizada también es apropiada para elaborar concreto; sin embargo, hay ocasiones en que esto no se cumple, porque hay aguas con pequeñas cantidades de azúcares que no afectan su potabilidad pero pueden hacerlas inadecuadas para la fabricación de concreto, en la medida que retardan o inhiben el fraguado del cemento. Considerar otro escenario pudiera ser más conveniente, es decir, que el agua para elaborar concretos no necesariamente requiere ser potabilizada, aunque sí debe satisfacer determinados requisitos mínimos de calidad, lo que abre las posibilidades para satisfacer la demanda de agua para la elaboración de mezclas de concreto de óptima calidad en lugares donde no hay un buen servicio de acueducto, pero sí un importante régimen de lluvias. De otra parte, la calidad de las aguas lluvia está directamente relacionada con la calidad del aire [2]; por eso, teniendo en cuenta esta premisa y sabiendo que el sector del barrio Robledo de Medellín, a la altura de la Institución Universitaria Colegio Mayor de Antioquia, es uno de los más contaminados en esta ciudad, según estudios del Área Metropolitana del Valle de Aburrá (AMVA) [3], se hace necesario realizar los análisis de calidad de las aguas lluvia en este punto, ya que será el material empleado en la elaboración de las muestras de la investigación.

Otro aspecto por tener en cuenta es el impacto ambiental que la actividad constructora genera sobre los hábitats urbanos y rurales, entre ellos el relacionado con el consumo de agua necesaria para la reacción del cementante en una mezcla de concreto [4]; y es que suele pensarse que en Medellín el agua potabilizada es abundante y fácil de obtener, pero la realidad es distinta, pues cerca del $95 \%$ del agua que se usa en hogares, colegios y obras de construcción proviene de fuentes que están ubicadas por fuera del Valle de Aburrá, por lo que cada vez es más difícil y costoso potabilizar este recurso, cuando en la mayoría de los casos se podrían emplear aguas reutilizadas o de lluvia. Eliminar el uso de agua potabilizada en actividades que no la requieren podría ahorrar hasta un $45 \%$ del consumo de este recurso en Medellín y el Valle de Aburrá [5]; entre estas actividades se encuentra la elaboración de estructuras de concreto, prefabricados y obras de infraestructura.

Sin duda, se hace necesario encontrar formas más reflexivas de llevar a cabo una actividad económica y social tan importante como es la construcción, pero, por más bien intencionadas que estas puedan ser, deben ser sometidas a estudios que minimicen la incertidumbre sobre aspectos relevantes como desempeño a exigencias externas, comportamiento físico-químico y estabilidad [6]. El estudio de estas variables y su discusión permitirán establecer un paradigma que, sin eludir las exigencias técnicas y económicas de la actividad edilicia, propugne garantizar hábitats construidos con una menor huella ecológica e hídrica sobre los territorios intervenidos.

\section{Materiales y MÉtodos}

Para el desarrollo de la investigación se escogió el campus de la Institución Universitaria Colegio Mayor de Antioquia, dada su proximidad a la estación de monitoreo de calidad del aire de ese sector de la ciudad (100 m), ubicada en la Facultad de Minas de la Universidad Nacional de Colombia (MED-UNFM), y que arroja una de las mayores contaminaciones de todos los puntos monitoreados por el AMVA, como se muestra en la Tabla I; aunque cabe aclarar que a nivel mundial aún está la discusión sobre el parámetro que dá lugar al término contaminado, pues se ha tomado como referencia el tamaño de partícula exclusivamente, y se hace necesaria una mirada desde la composición química del polvo o aerosol suspendido en la atmósfera [7].

\section{TABLA I \\ Material Particulado PM 10; Promedios} ANUALES

\begin{tabular}{|c|c|}
\hline Año & PM 10 $\boldsymbol{\mu g} / \mathbf{m}^{\mathbf{3}}$ \\
\hline 2012 & 76,00 \\
\hline 2013 & 57,10 \\
\hline 2014 & 61,90 \\
\hline
\end{tabular}

Fuente: Área Metropolitana del Valle de Aburrá, 2014. 
La Norma Nacional, Resolución 610 de 2010, establece que el límite para el promedio anual debe ser de $50 \mu \mathrm{g} /$ $\mathrm{m}^{3}$, y se observa que en los años monitoreados este sector de la ciudad lo excede [8].

\section{A. Muestras de agua}

Se instaló un sistema de recolección de aguas lluvia y se monitoreó durante seis meses la calidad del agua recolectada. El agua lluvia, depositada sobre la cubierta de la sala de profesores de la Facultad de Arquitectura e Ingeniería, se condujo a través de una tubería de PVC de 7,62 $\mathrm{cm}$ (tres pulgadas) de diámetro; luego, se almacenó en tanques plásticos de 1000 litros de capacidad.

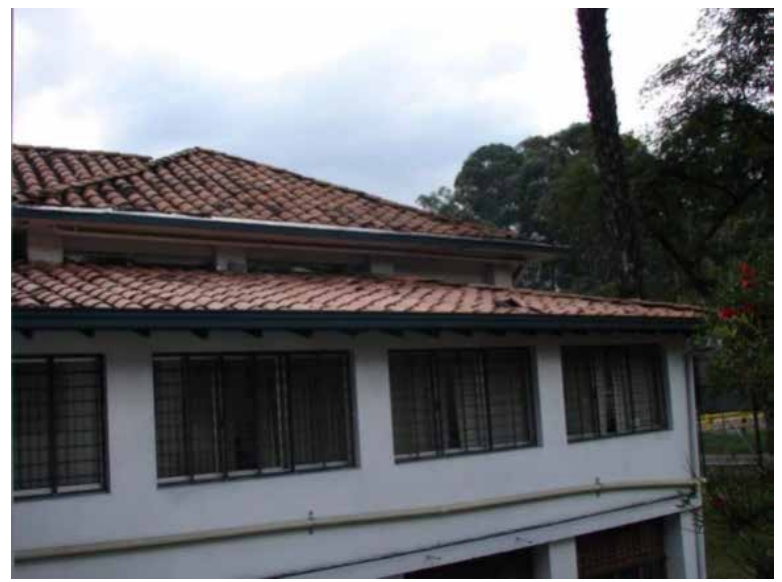

Fig. 1. Cubierta para captación de aguas lluvia. Fuente: Medina, $\mathrm{C}$.

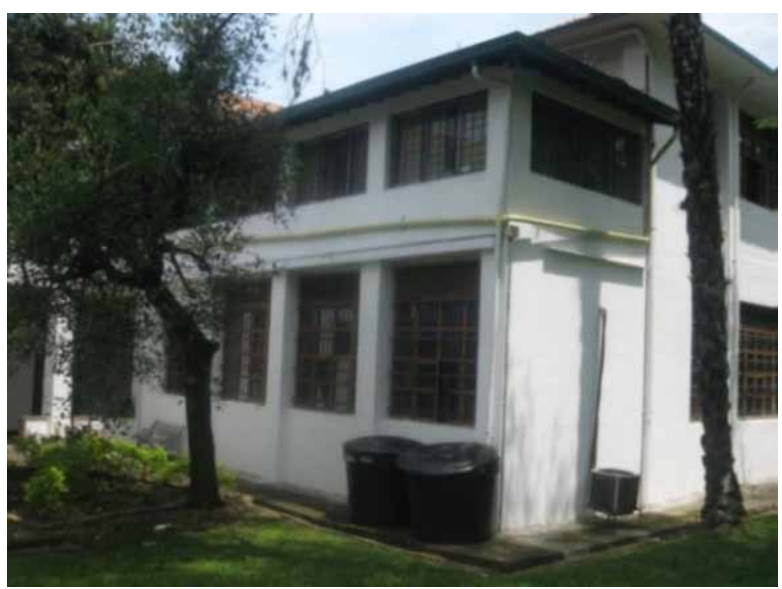

Fig. 2. Tuberías y tanques de almacenamiento del agua lluvia. Fuente: Medina, C.

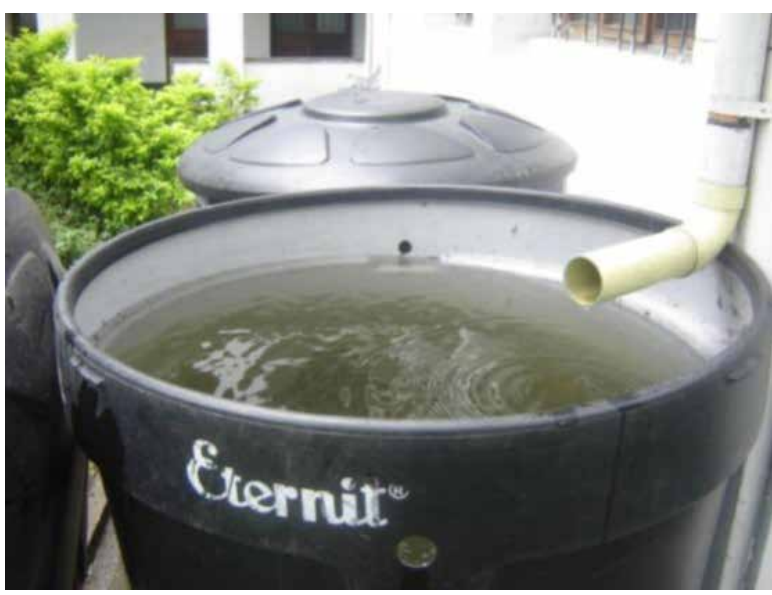

FiG. 3. Almacenamiento de aguas lluvia. Fuente: Medina, C.

A continuación se muestra el promedio histórico de los seis meses de caracterización del agua lluvia, recolectada en la zona de estudio afectada por la estación de monitoreo MED-UNFM.

\section{Tabla II}

DATOS OBTENIDOS DEL ANÁlisis DEL AGUA

LLUVIA POR PARTE DEL LABORATORIO DE CONTROL DE CALIDAD DE LA IUCMA

\begin{tabular}{|c|c|c|}
\hline Mes/Año & pH & $\begin{array}{c}\text { Turbiedad } \\
\text { NTU }\end{array}$ \\
\hline $09 / 2012$ & 6,30 & 2,18 \\
\hline $10 / 2012$ & 6,54 & 1,62 \\
\hline $11 / 2012$ & 6,39 & 2,22 \\
\hline $12 / 2012$ & 6,49 & 2,41 \\
\hline $03 / 2013$ & 6,12 & 2,40 \\
\hline $04 / 2013$ & 6,17 & 2,76 \\
\hline Promedio & 6,34 & 2,27 \\
\hline
\end{tabular}

Fuente: Medina, C. y Bedoya C., 2013.

Una vez se tuvo información estadística concluyente se procedió a seleccionar las muestras de agua tanto potabilizada como lluvia para proceder a la confección de las mezclas, las cuales fueron llevadas al laboratorio de aguas certificado de la I.U. Colegio Mayor de Antioquia (LACMA). Para el caso de las aguas lluvia se llevó a cabo el siguiente procedimiento: 
- El agua de los primeros cinco minutos de lluvia se descartó, dado que en esta se deposita el material particulado presente en la atmósfera.

- Luego de los cinco minutos iniciales se procedió a llenar los tanques.

- Apenas se llegó al nivel máximo de llenado, se tomaron las muestras de agua, con personal calificado del LACMA para evitar incertidumbres debidas a la carencia de un protocolo óptimo.

- El agua potabilizada se tomó directamente del acueducto abastecido por la empresa prestadora del servicio en la ciudad de Medellín (Tabla III).

\section{TABLA III}

DAtos OBTENIDOS DEL ANÁLISIS DEL AGUA POTABILIZADA POR PARTE DEL LABORATORIO DE Control de CALIDAd de la IUCMA

\begin{tabular}{|c|c|c|}
\hline Mes/Año & pH & $\begin{array}{c}\text { Turbiedad } \\
\text { NTU }\end{array}$ \\
\hline $04 / 2013$ & 6,80 & 0,10 \\
\hline
\end{tabular}

Fuente: Medina C. y Bedoya C., 2013.

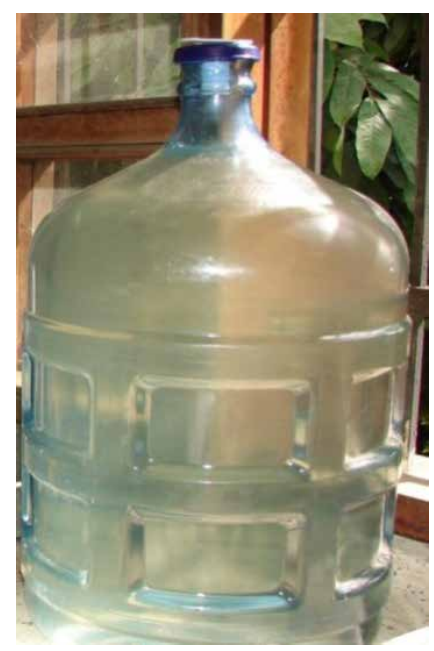

Fig. 4. Muestra de aguas lluvia enviada para caracterización a laboratorio. Fuente: Medina, C.

\section{B. Los agregados}

Los agregados provinieron del norte del Valle de Aburrá, zona donde se ubican la mayoría de canteras que abastecen de grava y arena a las empresas constructoras y productoras de concreto premezclado de Medellín y ciudades vecinas. Las características de estos agregados se muestran en la Tabla IV.

\section{TABLA IV}

AgREgAdOS EMPLEADOS EN LA INVESTIGACIÓN

\begin{tabular}{|l|c|c|}
\hline \multicolumn{1}{|c|}{ Característica } & $\begin{array}{c}\text { Agregado } \\
\text { grueso }\end{array}$ & $\begin{array}{c}\text { Agregado } \\
\text { fino }\end{array}$ \\
\hline Tamaño máximo & $19,05 \mathrm{~mm}$ & $\mathrm{NA}$ \\
\hline Módulo de finura & $\mathrm{NA}$ & 2,90 \\
\hline $\begin{array}{l}\text { Porcentaje de } \\
\text { absorción }\end{array}$ & 1,20 & 1,50 \\
\hline $\begin{array}{l}\text { Gravedad específica } \\
\text { aparente }\end{array}$ & $2750 \mathrm{~kg} / \mathrm{m}^{3}$ & $2732 \mathrm{~kg} / \mathrm{m}^{3}$ \\
\hline Granulometría & $\begin{array}{c}\text { Franja } \\
\text { NTC } 77\end{array}$ & $\begin{array}{c}\text { Franja NTC } \\
77\end{array}$ \\
\hline
\end{tabular}

Fuente: Medina C. y Bedoya C., 2013.

\section{Muestras de concreto}

El cemento usado fue Portland tipo III; con él se diseñaron dos mezclas de concreto para una resistencia al esfuerzo de compresión de $25 \mathrm{MPa}$ a 28 días, y con una relación agua/cementante de 0,60. Para efectos de comparación, la mezcla patrón o de referencia fue confeccionada con agua potabilizada. Se vaciaron muestras cilíndricas de $15,24 \mathrm{~cm}$. de diámetro por $30,48 \mathrm{~cm}$. de altura, determinando un juego de tres cilindros por cada fallado y por tipo de mezcla [9]; una vez desencofrados, se sometieron a inmersión en tanque de curado con agua saturada con cal. Se hicieron ensayos a edades de 1, 3, 7, 14, 28, 56 y 90 días. El asentamiento de las mezclas en estado fresco fue de $10,70 \mathrm{~cm}$. (4,21 pulg.) para el concreto con agua potabilizada, y de $10,50 \mathrm{~cm}$. (4,13 pulg.) para el concreto con aguas lluvia.

\section{TABla V}

MEZCla DE CONCRETO EMPLEADA EN LA INVESTIGACIÓN

\begin{tabular}{|l|c|}
\hline \multicolumn{1}{|c|}{ Material } & Partes \\
\hline Cemento & 1,00 \\
\hline Agregado fino & 2,61 \\
\hline Agregado grueso & 2,33 \\
\hline Rel. A/C & 0,60 \\
\hline
\end{tabular}

Fuente: Medina C. y Bedoya C., 2013. 


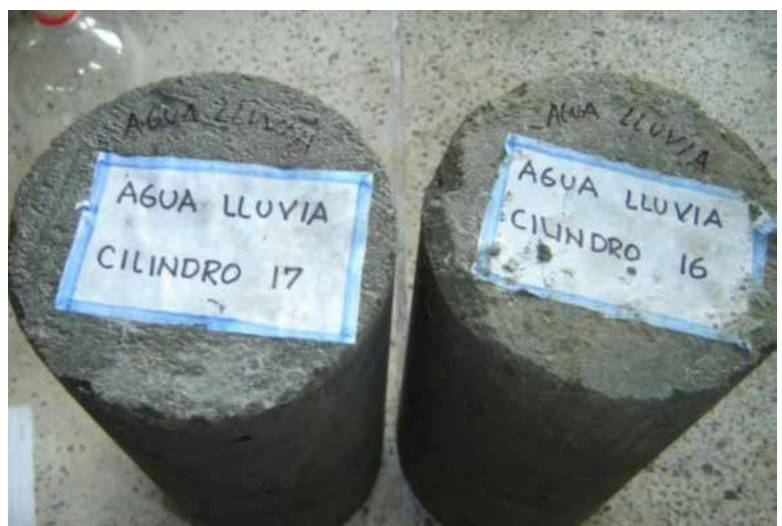

Fig. 5. Muestras cilíndricas de concreto confeccionados con aguas lluvia. Fuente: Medina, C.

A los cilindros ensayados a 28 días se les hicieron pruebas de microscopía de barrido electrónico (SEM), para estudiar su microestructura y sus componentes químicos por medio de sonda, con el fin de determinar la presencia de ciertos elementos en ambas mezclas y sus posibles incidencias en el comportamiento futuro del concreto endurecido.

\section{Resultados}

A continuación se muestran los resultados obtenidos de los distintos ensayos de caracterización de las materias primas y de las pruebas al concreto endurecido.

\section{A. De las muestras de agua}

Según la NTC 3459, el agua para confeccionar mezclas de concreto debe cumplir con los parámetros que se muestran en la Tabla VI.

\section{Tabla VI}

ParÁmetros límite del AGUA PARA CONFECCIÓN DE CONCRETO

\begin{tabular}{|c|c|}
\hline Parámetro & Concentración $\mathbf{( m g / L )}$ \\
\hline Sulfatos & 1000 \\
\hline Cloruros & 1000 \\
\hline Sólidos totales & 50000 \\
\hline Sólidos disueltos & 2000 \\
\hline $\mathrm{pH}$ & $\geq 5$ \\
\hline Turbiedad & NA \\
\hline
\end{tabular}

Fuente: NTC 3459.
Las muestras de aguas lluvia y agua potabilizada fueron analizadas bajo los parámetros exigidos por la NTC 3459. Dichas muestras fueron recolectadas el mismo día (23 de abril de 2013) para tener posibilidad de trazabilidad, en caso de requerir una posterior comparación de resultados o solicitud de datos a la empresa prestadora de servicios públicos de acueducto y alcantarillado de la ciudad de Medellín. Los resultados se muestran en la Tabla VII.

\section{Tabla VII}

COMPARACIÓN DE PARÁMETROS LÍMITE ENTRE AGUA POTABILIZADA Y AGUAS LLUVIA PARA CONCRETO

\begin{tabular}{|c|c|c|c|}
\hline Parámetro & $\begin{array}{l}\text { Aguas } \\
\text { Lluvia } \\
\text { (mg/L) }\end{array}$ & $\begin{array}{c}\text { Agua } \\
\text { Potabilizada } \\
(\mathrm{mg} / \mathrm{L})\end{array}$ & $\begin{array}{c}\text { NTC } 3459 \\
(\mathrm{mg} / \mathrm{L})\end{array}$ \\
\hline Sulfatos & 5,00 & 12,00 & 1000 \\
\hline Cloruros & 16,30 & 19,50 & 1000 \\
\hline $\begin{array}{l}\text { Sólidos } \\
\text { totales }\end{array}$ & 28,50 & 32,00 & 50000 \\
\hline $\begin{array}{c}\text { Sólidos } \\
\text { disueltos }\end{array}$ & 27,00 & 1,00 & 2000 \\
\hline $\mathrm{pH}$ & 5,10 & 6,80 & $\geq 5$ \\
\hline Turbiedad & $4,20 \mathrm{NTU}$ & $0,10 \mathrm{NTU}$ & NA \\
\hline
\end{tabular}

Fuente: NTC 3459.

Como se puede observar, según los resultados emitidos por el laboratorio, en ambos casos el agua es apta para la confección de una mezcla de concreto.

\section{B. Datos del concreto endurecido}

En la Tabla VIII se muestran los resultados de los ensayos para determinar la resistencia al esfuerzo de la compresión de las mezclas de concreto con agua potabilizada y con aguas lluvia. 


\section{TABLA VIII}

RESISTENCIA AL ESFUERZO DE LA COMPRESIÓN

\begin{tabular}{|c|c|c|}
\hline Días & $\begin{array}{c}\text { F'c Agua } \\
\text { potabilizada } \\
\text { (MPa) }\end{array}$ & $\begin{array}{c}\text { F'c Aguas } \\
\text { lluvia } \\
\text { (MPa) }\end{array}$ \\
\hline 1 & 7,24 & 7,62 \\
\hline 3 & 16,84 & 17,34 \\
\hline 7 & 22,06 & 23,76 \\
\hline 14 & 24,70 & 24,05 \\
\hline 28 & 27,74 & 27,51 \\
\hline 56 & 28,75 & 29,18 \\
\hline 90 & 33,15 & 34,13 \\
\hline
\end{tabular}

Fuente: Medina C. y Bedoya C., 2013.

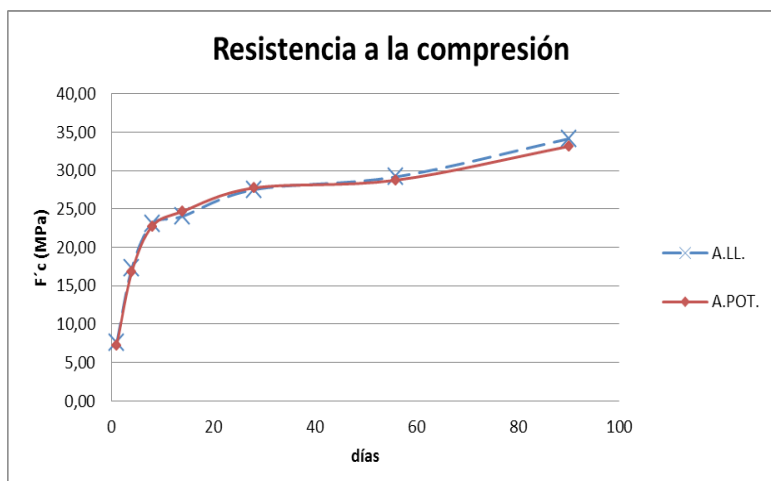

Fig. 6. Evolución de la resistencia al esfuerzo de la compresión hasta los 90 días. Fuente: Medina C. y Bedoya C., 2013.

Las Tablas IX y X muestran las estadísticas de los ensayos y sus respectivos resultados.

\section{TABLA IX}

ESTADÍSTICAS DE LOS ENSAYOS A COMPRESIÓN DEL CONCRETO CON AGUAS LLUVIA

\begin{tabular}{|c|c|c|c|}
\hline $\begin{array}{c}\text { Edad de } \\
\text { fallado }\end{array}$ & Media & $\begin{array}{c}\text { Desviación } \\
\text { estándar }\end{array}$ & $\begin{array}{c}\text { Coeficiente de } \\
\text { variación }\end{array}$ \\
\hline 1 & 7,62 & 0,39 & $5,13 \%$ \\
\hline 3 & 17,34 & 0,17 & $0,95 \%$ \\
\hline 7 & 23,06 & 0,75 & $3,25 \%$ \\
\hline 14 & 24,05 & 0,18 & $0,74 \%$ \\
\hline 28 & 27,51 & 0,18 & $0,64 \%$ \\
\hline 56 & 29,18 & 0,94 & $3,21 \%$ \\
\hline 90 & 34,13 & 0,44 & $1,30 \%$ \\
\hline
\end{tabular}

Fuente: Salazar A., Neira G. y Medina C. 2013.

\section{TABLA X}

ESTADÍSTICAS DE LOS ENSAYOS A COMPRESIÓN DEL CONCRETO CON AGUA POTABILIZADA

\begin{tabular}{|c|c|c|c|}
\hline $\begin{array}{c}\text { Edad de } \\
\text { fallado }\end{array}$ & Media & $\begin{array}{c}\text { Desviación } \\
\text { estándar }\end{array}$ & $\begin{array}{c}\text { Coeficiente de } \\
\text { variación }\end{array}$ \\
\hline 1 & 7,24 & 0,14 & $1,98 \%$ \\
\hline 3 & 16,84 & 0,02 & $0,10 \%$ \\
\hline 7 & 22,76 & 0,09 & $0,39 \%$ \\
\hline 14 & 24,70 & 0,13 & $0,54 \%$ \\
\hline 28 & 27,74 & 0,10 & $0,38 \%$ \\
\hline 56 & 28,75 & 0,04 & $0,14 \%$ \\
\hline 90 & 33,15 & 0,98 & $2,97 \%$ \\
\hline
\end{tabular}

Fuente: Salazar A., Neira G. y Medina C. 2013.

\section{Microscopía}

Una vez se hicieron los ensayos al esfuerzo de compresión, se tomaron muestras de ambos concretos para ser llevados al laboratorio de microscopía avanzada, con el fin de determinar su comportamiento en cuanto a microestructura y elementos químicos presentes en la pasta de cemento. Las muestras obedecían a probetas cilíndricas de 28 días de curado en agua saturada con cal.

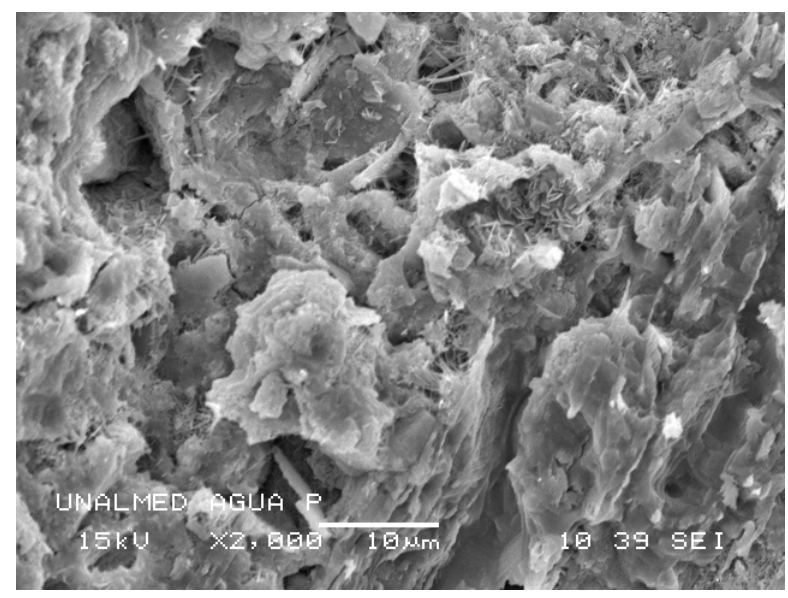

Fig. 7. Microscopía de agua potabilizada; $2000 \mathrm{X}$. Fuente: Medina C. y Bedoya C., 2013. 


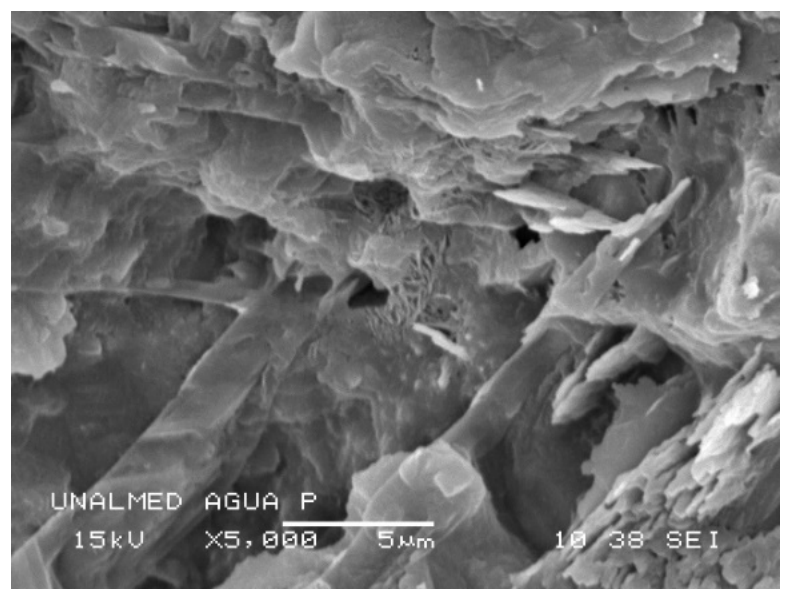

Fig. 8. Microscopía de agua potabilizada; $5000 \mathrm{X}$. Fuente: Medina C. y Bedoya C., 2013.

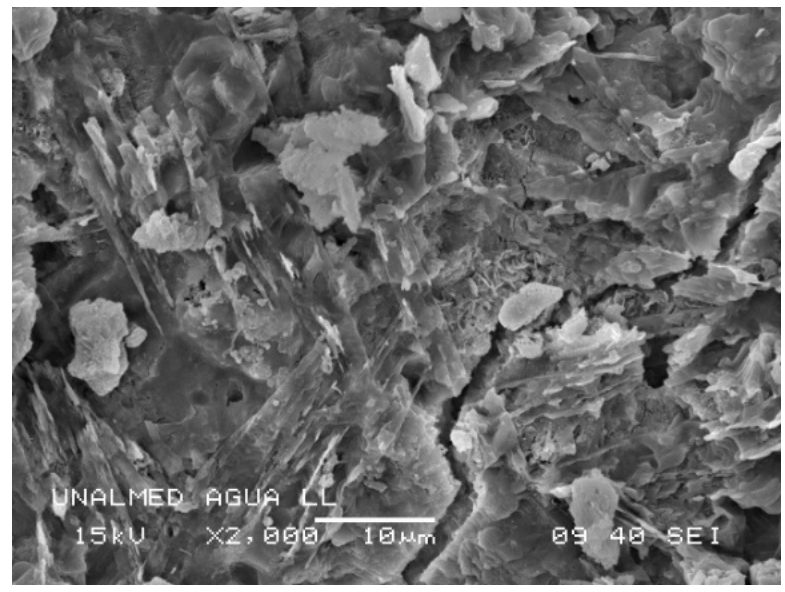

Fig. 9. Microscopía de agua lluvia; 2000 X. Fuente: Medina C. y Bedoya C. 2013.

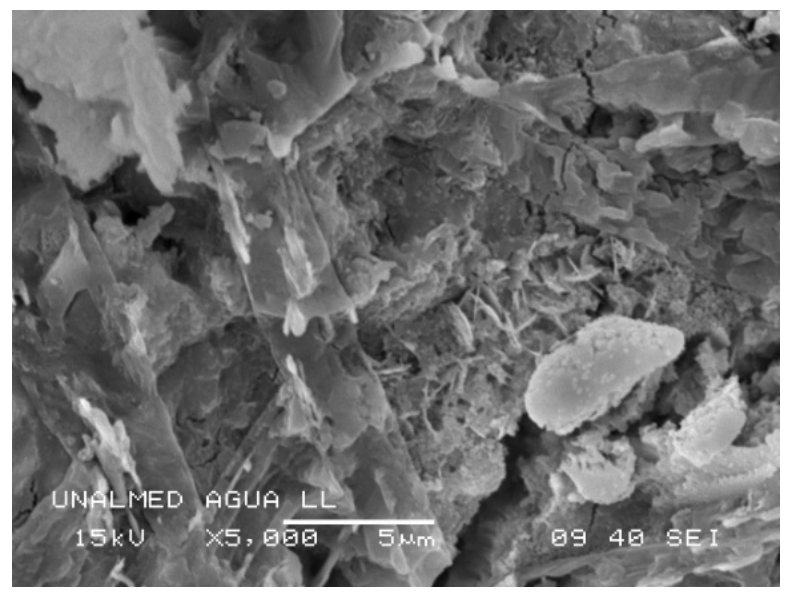

Fig. 10. Microscopía de agua lluvia; 5000 X. Fuente: Medina C. y Bedoya C. 2013.

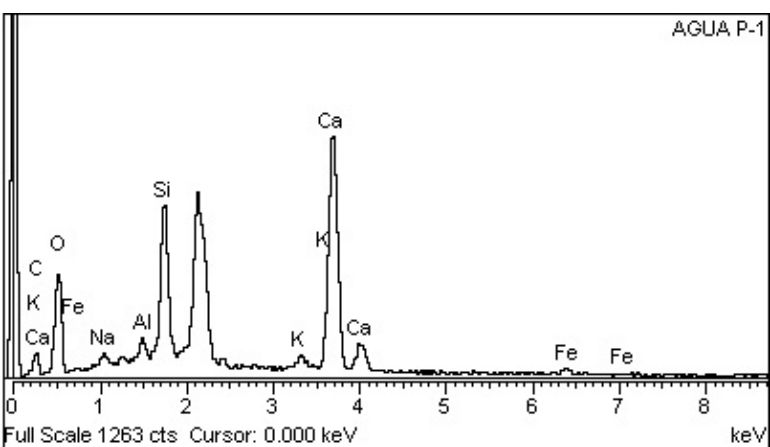

Fig. 11. Análisis químico por microscopía de barrido electrónico; agua potabilizada. Fuente: Medina C. y Bedoya C. 2013.

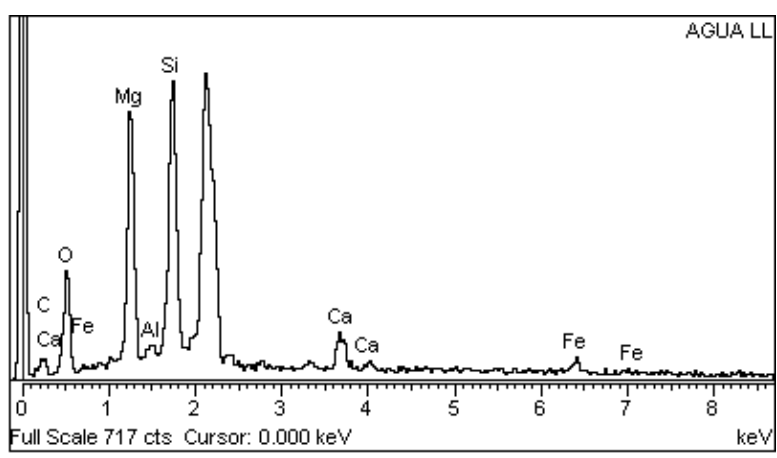

Fig. 12. Análisis químico por microscopía de barrido electrónico; agua lluvia. Fuente: Medina C. y Bedoya C. 2013.

\section{Conclusiones}

El agua lluvia empleada en la presente investigación no afectó negativamente el comportamiento del concreto desde el punto de vista físico-mecánico. Estadísticamente hablando, las resistencias al esfuerzo delacompresión obtenidas a distintas edades soniguales en ambas mezclas. Se recomienda complementar este estudio con ensayos de comportamiento en cuanto a módulo de elasticidad.

$\mathrm{Si}$ bien la relación $\mathrm{A} / \mathrm{C}$ estuvo alta $(0,60)$, las resistencias obtenidas estuvieron en los rangos del diseño de mezclas; sin embargo, se recomienda trabajar con relaciones $\mathrm{A} / \mathrm{C}$ menores, en aras de una mayor durabilidad del concreto endurecido. En este trabajo se analizó especialmente la resistencia al esfuerzo de la compresión.

Aunque la variable estudiada fue el agua lluvia, es de anotar que el comportamiento del concreto fresco fue idéntico para ambas mezclas en cuanto a características tales como el asentamiento, la trabajabilidad y la 
apariencia superficial. También se encontró que el color del concreto no se modificó, a pesar de que la muestra de agua lluvia presentaba un tono más turbio que la muestra del agua obtenida del acueducto.

Si bien en la ciudad de Medellín se presentan zonas con distintas concentraciones de material particulado y gaseoso, es positivo reseñar que en la presente investigación se ha tomado como punto de referencia uno de los más contaminados, según información oficial del AMVA - Red de monitoreo de la calidad del aire-, permitiendo inferir que es posible que las aguas lluvia del Valle de Aburrá sean aptas para ser empleadas en la confección de concretos y morteros. Sin embargo, se hace necesario continuar con la investigación, tomando muestras en dos puntos distintos al estudiado; en tal sentido, captar aguas lluvia en las zonas norte y sur, permitiendo así una posterior correlación de resultados a nivel de la región metropolitana.

\section{Agradecimientos}

Los autores agradecen al personal del laboratorio de Microscopía Avanzada de la Universidad Nacional de Colombia, Sede Medellín; del laboratorio de Desarrollo de Materiales de la empresa Ecoingeniería S.A.S., y del laboratorio de materiales de la Institución Universitaria Colegio Mayor de Antioquia, por su apoyo en el desarrollo de esta investigación. También expresan sus agradecimientos a la Dirección de Investigación y Extensión de la Facultad de Arquitectura de la Universidad Nacional de Colombia, Sede Medellín, por el apoyo brindado en movilidad para la exposición de este trabajo en eventos regionales e internacionales.

\section{REFERENCIAS}

[1] ICONTEC. Norma Técnica Colombiana NTC 3459: Agua para la elaboración de concretos. Bogotá D.C., Icontec, 2001.

[2] J. Arroyave. "Evaluación económica de la captación de agua lluvia como fuente alternativa de recurso hídrico en la Institución Universitaria Colegio Mayor de Antioquia". Medellín, Revista de Producción Más Limpia, Vol. 6., N. ${ }^{\circ}$ 1, pp. 76-84, 2011.

[3] AMVA; Procuraduría. Informe de monitoreo de calidad del aire en el Valle de Aburrá. Medellín: Ed. Área Metropolitana del Valle de Aburrá, 2010.

[4] C. Bedoya. Construcción sostenible, para volver al camino. Medellín: Ed. DIKÉ-Cátedra UNESCO de Sostenibilidad, 2011.

[5] AMVA. Documento base para la formulación de una política pública de construcción sostenible en el Valle de Aburrá. Medellín: Ed. Área Metropolitana del Valle de Aburrá, 2010.

[6] A. Salazar. Impacto ambiental de los materiales de construcción en Colombia. Estudio de Caso ciudad de Cali. Bogotá: Ed. UPME, 2012.

[7] I. Schifter. La huella invisible: humos, polvos $y$ perfumes. México, D.F.: Fondo de Cultura Económica, 2009.

[8] AMVA. Red de calidad del aire del Valle de Aburrá. Medellín, 2014. Recuperado de: http://www.metropol.gov.co/CalidadAire/ isodocRedAire/Resumen $\% 20$ anual $\% 20$ calidad\%20del\%20aire\%202014.pdf 